

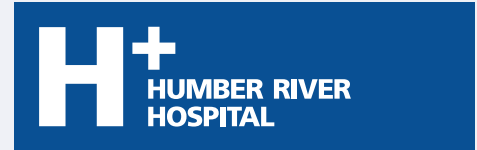

HUMBER RIVER HOSPITAL is one of Canada's largest community acute care hospitals, serving a population of more than 850,000 people in the northwest Greater Toronto Area.

The multi-site hospital currently operates out of its Wilson Avenue acute care site and Finch and Church Street reactivation care centres with a total of 722 beds, just over 3,800 employees, approximately 700 physicians and over 1,000 volunteers.

Affiliated with the University of Toronto and Queen's University, Humber River Hospital is North America's first fully digital hospital. Part of Humber River Hospital's digital infrastructure includes completely automated laboratory services, robots sorting and mixing medications, electronic health records, tracking systems for patients undergoing surgery that update families through their cellphones and patient computer bedside terminals - all varieties of technologies that automate information, eliminate paper and provide a connected experience for patients, staff and families.

Humber River Hospital was awarded Accreditation with Exemplary Standing in 2018 and since its opening in 2015 has received numerous awards and accolades for technological advancements and innovation (www.hrh.ca).

\title{
Nursing Leadership in the Fully Digital Practice Realm
}

Vanessa Burkoski, RN, BScN, MScN, DHA

Chief Nursing Executive \& Chief, People Strategy

Humber River Hospital

Toronto, ON

\begin{abstract}
In the fully digital practice realm, a new relationship has emerged between nurses, patients and technology. Nursing leadership in this unique practice setting requires new and enhanced skills, knowledge and abilities. Maintaining the principles of patient and family centredness, attentiveness to nurses' concerns regarding workflows and involvement of nurses in the design of technologies is critical for creating a safe, professional practice environment in the fully digital hospital. Technology cannot replace the humanness of caring. Mindfulness of the digital impact on the therapeutic relationship is necessary. Competency in technology is the new proficiency that nursing leaders will need to acquire.
\end{abstract}

By leveraging technology, Toronto's Humber River Hospital (HRH) is aiming to achieve high reliability, a key strategic direction to consistently deliver the safest, highest quality care alongside compassion, professionalism and respect - the core values of the hospital. Across healthcare organizations, many improvements in patient safety have been enabled through access to better information. To my 
knowledge, nowhere else in North America is a hospital so rich in usable data that can influence ongoing improvements in the safety and quality of care delivery. The fully digital infrastructure at HRH provides the opportunity for monitoring and analysis of patient outcomes and organizational processes, which has fostered a robust culture of continuous quality improvement.

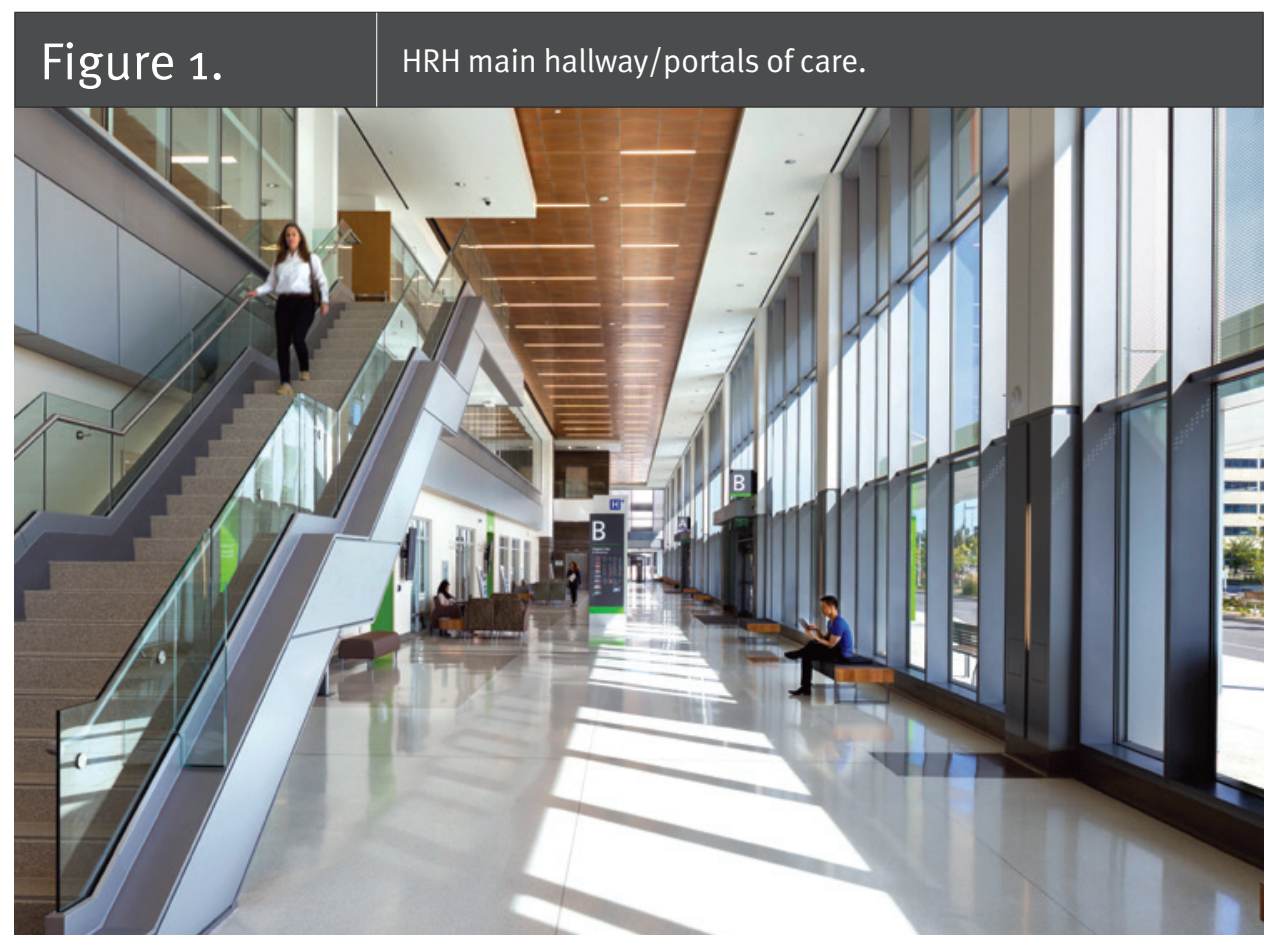

\section{Transforming Care}

So what is the impact of all this digital infrastructure on nursing practice and patient outcomes? There is clearly a responsibility to examine, explore and investigate whether this unique hospital environment is accomplishing its goals (Figure 1). This special edition of the Canadian Journal of Nursing Leadership will share the results of our foundational research, largely examining the intersection of clinical practice and technology from the nurse perspective, as well as one study that includes the patient perspective. We focused our studies on the digital systems and devices that are fundamental to nursing care delivery and might pose the greatest risk to safety and patient experience. For example, the closed-loop medication system (Figure 2), smartphone communication technology, integrated bedside terminal and violence prevention technology were examined to gain a better understanding of the impact of these technologies on nursing practice and patient satisfaction. We also wanted to obtain the perspectives of nurses on their adoption of technology. This was important to us because a digital infrastructure 


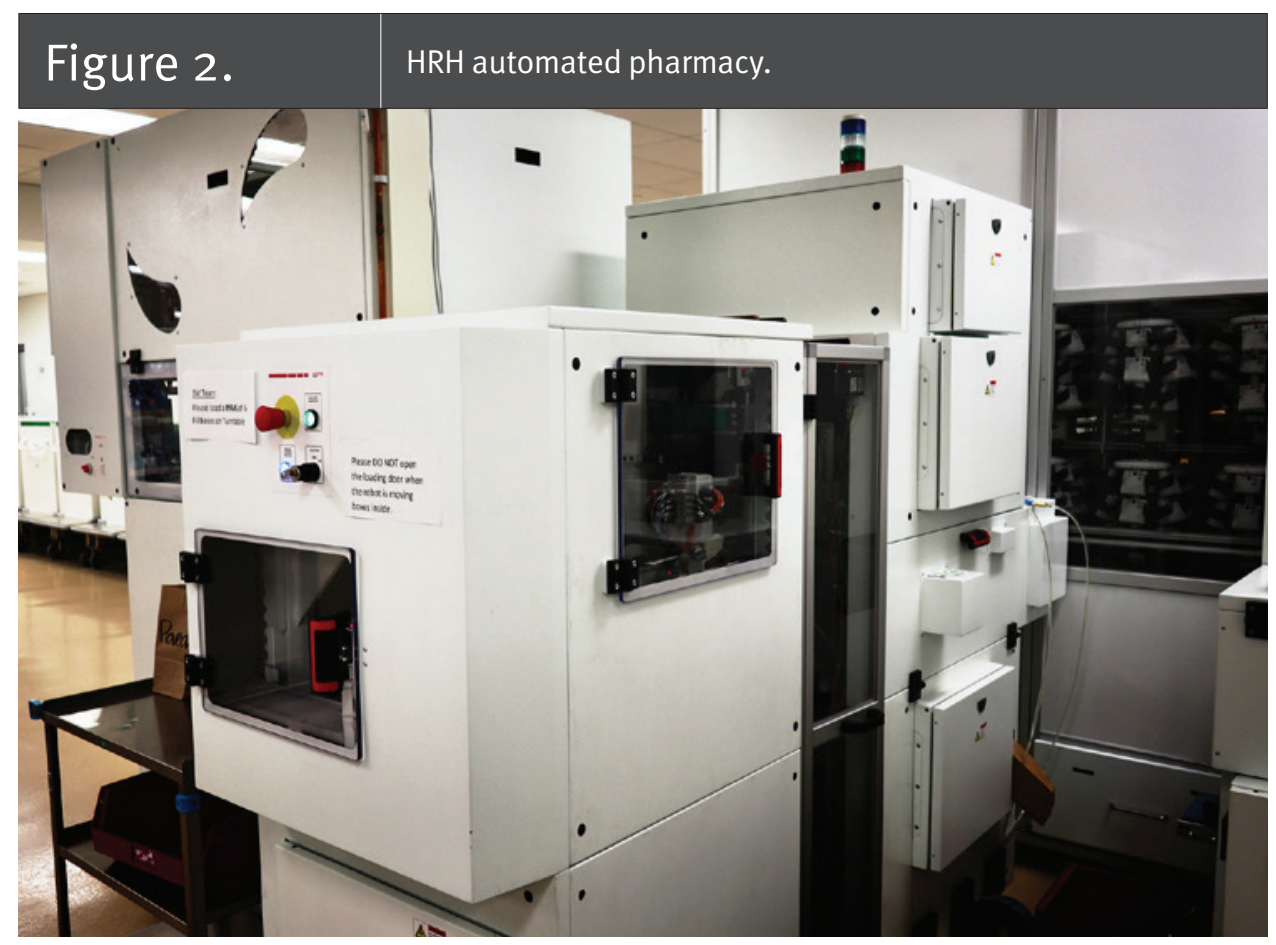

is of limited value if the end-users are compromised in its adoption. In these studies, we explored nurses' experience of practicing in a fully digital hospital and generational differences in technology adoption. Finally, we provide a discussion paper that describes how HRH has leveraged the electronic medical record (EMR) to increase evidence-based practice integration and improve patient outcomes. The questions we have posed in these studies are just a start. Nursing leaders will need to ask many more important questions over time to steer the integration of technology in a direction that will continue to elevate nursing practice and safeguard the patient experience.

Findings from our studies revealed that the technology at HRH is already having a positive impact on how nurses practise and transform care. One key benefit of the HRH fully digital environment is the ability to provide direct-care nurses and clinical leaders with point-in-time information that can be used to monitor, guide and improve care delivery. At HRH, nursing documentation in the EMR is built to the specifications of the Registered Nurses' Association of Ontario's (RNAO) best practice guidelines, which not only supports a reduction in practice variability but also enables nursing-sensitive indicators to be measured against patient outcomes. Electronic reminders in the EMR improve adherence to evidence-based practice, and data collected through the EMR show nurses which interventions are working or not. Using nursing-sensitive data, leaders can truly identify the value that nursing brings to the optimization of patient health outcomes. 
At HRH, among our nursing leaders, I have come to appreciate the intimate nature of the relationship between nurses, patients and technology. An example of this relationship often occurs when nurses are conducting their initial clinical assessments. Rather than having to ask patients to repeat their histories yet again, nurses in the digital workplace find that all the required information already exists in the patient's chart. In this case, sometimes patients may feel ignored or perceive that nurses are distracted by the technology. On the other hand, a great example of how technology is serving patients and staff well at $\mathrm{HRH}$ has been embedded in the perioperative program. The STERIS RealView patient tracking system is used to monitor surgical patients throughout their perioperative journey. Typically, in a non-digital environment, patients are separated from family and friends to be prepared for their operations. This period of time typically incites a great deal of anxiety for both patients and families. The STERIS RealView surgical patient tracking system at HRH connects care providers and families in real time through frequent communication notifications sent via smartphone or display boards, continually informing families of their loved one's status and progression through the surgical journey, even at home or out of the country (see Figure 3). This has had a significant impact on reducing anxiety for patients and families while fostering positive experiences. The tracking system is also used by HRH staff to communicate between departments, enabling each perioperative area to predict patient flow and guide resource allocation decisions. The tracking system highlights how digital infrastructure has been integrated to support nursing communication and decision making, with concurrent engagement of patients and families. Nurse leaders have an important role to play in advocating for technical solutions that maintain the principles of patient and family centredness through timely and accessible information exchange.

\section{Reverence for End-Users}

Understanding the experience of nurses practising in the digital environment is crucial to ensure that technology does not impair critical thinking or erode the nursing process. A clear example is the manner in which vital signs are captured and displayed in the EMR. When vital signs are assessed, the workflow for 


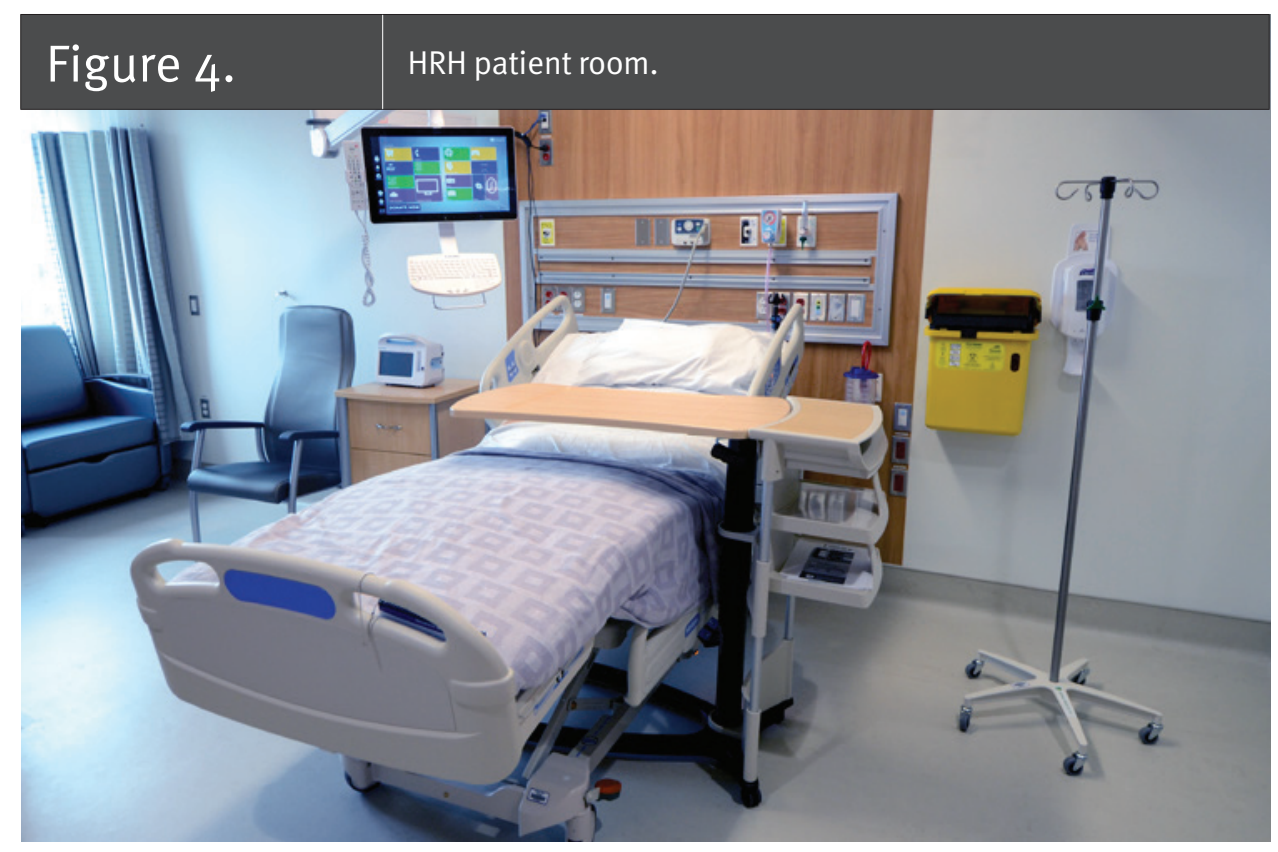

electronic documentation should align with the nursing process and the manner in which the data are presented must support the identification of changes and trends in patients' health status over time. Several nurses at HRH raised the concern that the graphic representation of vital signs in the EMR "is hard to read," and all nurses we talked to expressed a preference toward the tabular display of vital signs. Following a heuristic evaluation of how vital signs were captured and presented in the EMR, two key issues emerged. First, variability existed in the level of automation and collection of vital signs. Second, the design of the vital signs display did not make the most of the opportunity to support nurses' identification of trends that might signal patient deterioration. Nurses at the front line of care are in the best position to recognize opportunities for improvement because they are closest to the work. For nurse leaders, sensitivity to operations through attentiveness to front-line nursing concerns is essential for successful integration of technology.

Integrating the EMR into nursing practice is not easy. Nursing leaders need to recognize that technology changes the way care is delivered. One essential strategy for nurse leaders is to have the end-users, nurses, involved in the design of technologies to create systems that complement nursing practice and avoid the conditions that necessitate the development of workarounds. Nurse leaders will have to intervene wherever necessary to maximize the integration of technology that can be used effectively in the context of professional care and minimize the potential of nurses having to compensate for poorly designed systems. Technology should be leveraged to augment nursing care, creating a safer, more effective and efficient practice environment as much as technology supports patients and families with their informational needs (see Figure 4). 
One of the major benefits of EMR integration is that it allows nurse leaders the ability to work with the front line to review real-time data, assess and address current challenges and explore potential efficiencies. At HRH, this work is accomplished in an electronic test world that models the live EMR. With this capability, front-line users are able to co-create and test solutions that enhance nursing efficiencies, capture data and optimize patient safety. The ability to build and test solutions by deferring to the experts, in this case nurses, is a hallmark of highreliability hospitals. Nursing leaders in this environment must understand the principles of nursing informatics, electronic documentation, quality assurance and quality management to lay the foundation for engaging staff. Thus, nurse leaders will need to be mindful of the importance of front-line engagement in the development and design of digital technologies to create systems that work and garner the trust and confidence of nurses.

\section{Nursing Leadership in the New Digital Landscape}

New skills are required for nurses to become digitally literate. Nurses need to learn not only how to navigate the technology but also how to use the data and information that are contained in various formats within the technology. Education is essential, and leaders must ensure that dedicated time is carved out for nurses to concentrate on learning the technology. This learning cannot be achieved in the midst of trying to care for patients.

Although data analytics has great potential to improve decision making and care delivery, nursing leaders must be cautious about overloading nurses with digital technologies that may detract from their core responsibility of caring for patients using the nursing process. A requisite of nursing leaders will be to distinguish between technology that enables nurses to deliver better care and technology that burdens nurses in delivering care. When there are too many red dots on the control panel, none of them become the priority. As in the digital environment, when nurses are required to input too many variables or respond to multiple alarms, then none of them become relevant to care. Alarm fatigue in the fully digital environment is a problem that is amplified because of the integrated technology requiring nurse leaders to consider this impact on nursing care delivery. The right balance between nursing notifications that support harm reduction and error prevention and alarm fatigue is difficult to achieve.

Health technology is a professional issue in nursing that impacts practice, care delivery, education and research. Our studies, like others in the literature, alluded to a faint but existing tension between the nursing philosophy of caring and technology integration. Nursing is a uniquely human profession with caring as its central focus. Technology cannot replace the human touch. Just as current 
generations grapple with how smart technology has impacted families at the dinner table, nurse leaders will need to understand the perspective of nurses and patients regarding the impact of technology on delivering and receiving care. Otherwise, meeting the patients' spiritual, psychological and social needs (the art of nursing), may not be achieved appropriately within the digital landscape. The introduction of new and evolving technology requires nurse leaders to consider the impact of digitalization on the therapeutic relationship and to ensure that the patient, rather than the technology, remains the central focus.

Healthcare technology is going to take nursing to a new level. At HRH, technology is fostering better communication between nurses, patients, families and the interprofessional team. Some of the intricacies in the EMR touch not only on the importance and opportunity for interoperability between the different technologies (EMR, smartphones, integrated bedside terminal) but also on the nature of interprofessional collaboration. For example, drug libraries on SMART pumps are traditionally governed by pharmacy practice. Becoming fully digital required integration of pharmacy and nursing practice to create the EMR with appropriate inputs, outputs and documentation. This is an essential element in achieving the safest patient care. HRH is already achieving exceptional outcomes that are being sustained over time, such as zero medication errors, lowest percentage incidence of pressure injuries in Canada and highest rated hospital experience in the Central Local Health Integration Network. Technology is being leveraged at HRH so that nurses can accomplish even more in relation to higher quality patient outcomes. At the present time, the fully digital environment at HRH is unique. That won't last long because many other healthcare organizations are on the journey to becoming entirely digital. As chief nursing executive at HRH, I have been stretched to move in a direction that was foreign and uncomfortable. Technological competency was not on my list of most important nursing competencies before I came to HRH. What I have discovered over the past two years is that technology integration requires a consistent process of designing, testing, building, planning, educating and implementing. And once implementation of technology has occurred, there is another process of reflection, evaluation, revision and enhancement that is required to ensure that the system is right for nurses and right for patients. We are pleased to share the findings of our research during this distinctive point in time as North America's first fully digital hospital. Our hope is that these findings will assist others with developing the technological infrastructure to cultivate digitally capable nurses, now and into the future.

Correspondence may be directed to: Dr. Vanessa Burkoski, Humber River Hospital, 1235 Wilson Avenue, Toronto, ON, M3M 0B2; E-mail: vburkoski@hrh.ca. 


\section{$\mathbf{H}_{\substack{\text { Hosmegra neve } \\ \text { Hosith }}}^{+}$}

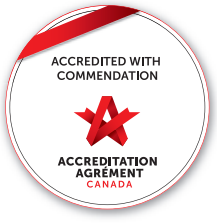

DOO
$\mathbf{D}$ BEST PRACTICE
SPOTLIGHT
ORGANIZATION
C A N A D A A
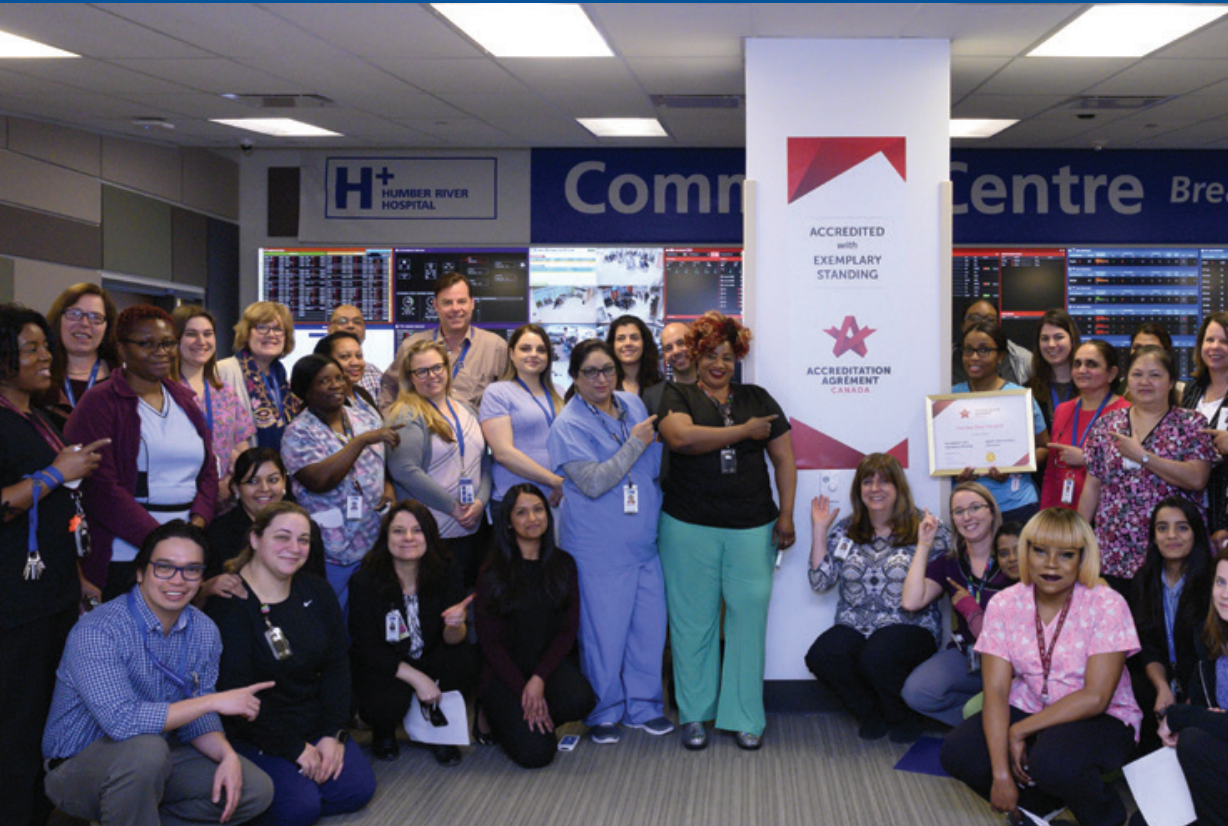

ACCREDITED

entre Breakthrough Intelligence

STEMPLARY

* cactorernon
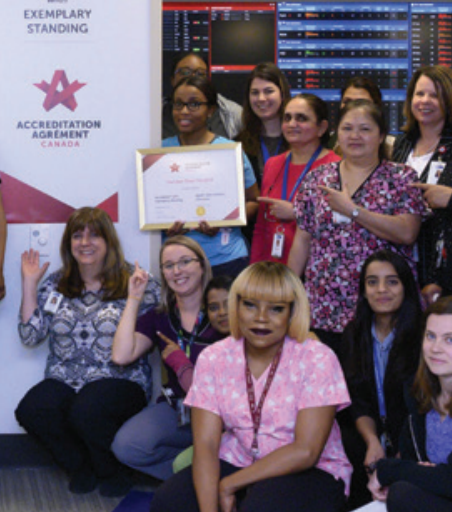

$\sin 2$
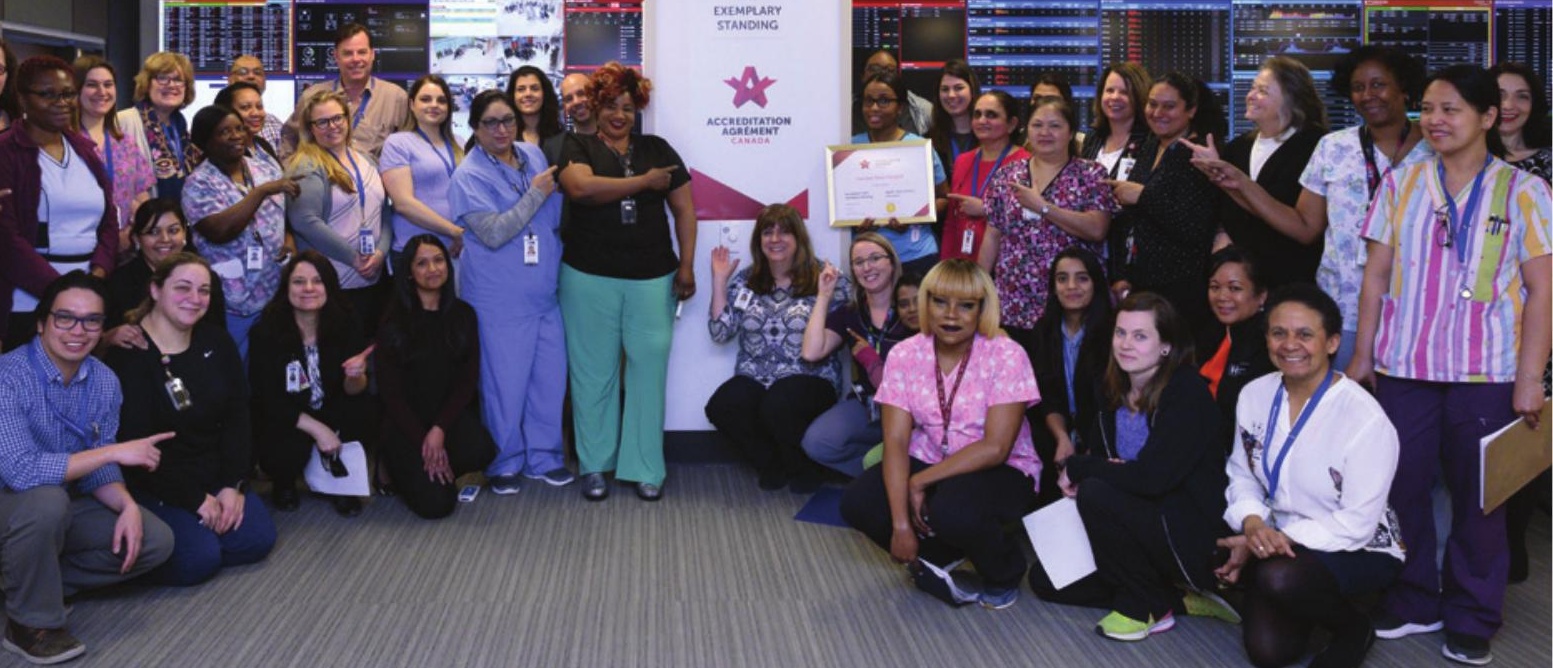

\section{Leading technology \& innovation integration in nursing}




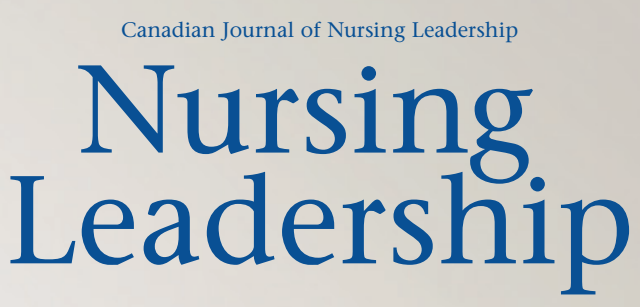

Leadership in Nursing Management, Practice, Education \& Research

NURSING LEADERSHIP - PERSPECTIVES AND INSIGHTS

Building Healthcare Leadership Capacity: Strategy, Insights and Reflections 10

The Role of Education in Developing Leadership in Nurses

Engaging Nurses in Future Management Careers: Perspectives on Leadership and Management Competency Development through an Internship Initiative 36

Black Nurse Leaders in the Canadian Healthcare System 50

Politics • Policy • Theory • Innovation

Us

ACEN
ACDSI

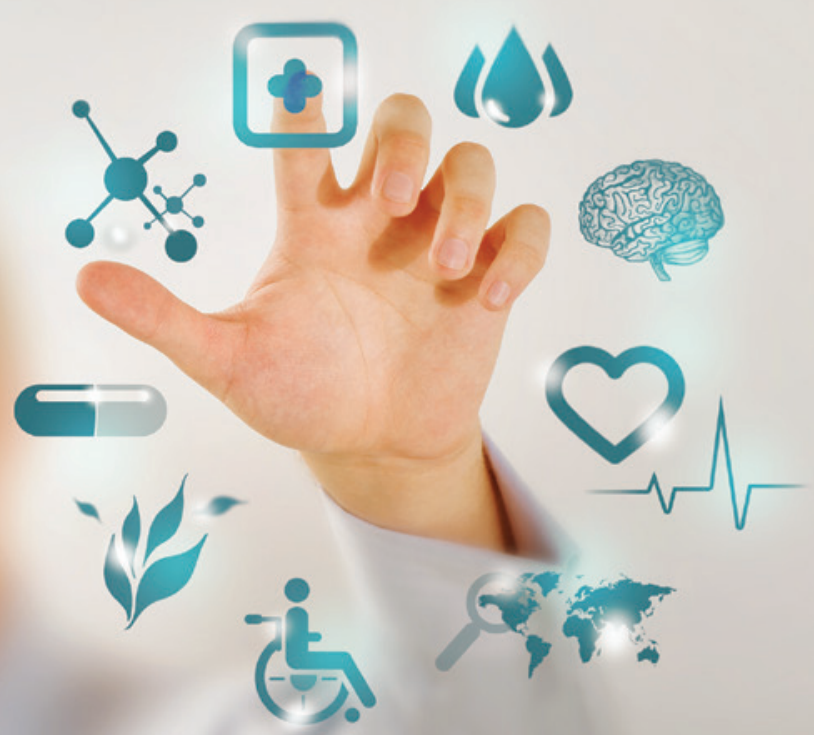

Covering politics, policy, theory and innovations that contribute to leadership in nursing administration, practice, teaching and research. 